\title{
Molecular population genetics and gene expression analysis of duplicated CBF genes of Arabidopsis thaliana
} Yen-Heng Lin ${ }^{1}$, Shih-Ying Hwang ${ }^{2}$, Po-Yen Hsu ${ }^{1}$, Yu-Chung Chiang ${ }^{3}$, ChunLin Huang ${ }^{1}$, Chun-Neng Wang ${ }^{4}$ and Tsan-Piao Lin*1

\author{
Address: ${ }^{1}$ Institute of Plant Biology, National Taiwan University, Taipei 10617, Taiwan, ${ }^{2}$ Department of Life Sciences, National Taiwan Normal \\ University, Taipei 10610, Taiwan, ${ }^{3}$ Department of Life Science, Pingtung University of Science and Technology, Pingtung 912, Taiwan and \\ ${ }^{4}$ Department of Life Sciences, National Taiwan University, Taipei 10617, Taiwan \\ Email: Yen-Heng Lin - b89206032@ntu.edu.tw; Shih-Ying Hwang - hsy9347@ntnu.edu.tw; Po-Yen Hsu - r94b42016@ntu.edu.tw; Yu- \\ Chung Chiang - yucchiang@hotmail.com; Chun-Lin Huang-d92b42001@ntu.edu.tw; Chun-Neng Wang - leafy@ntu.edu.tw; Tsan- \\ Piao Lin* - tpl@ntu.edu.tw \\ * Corresponding author
}

Published: 7 November 2008

BMC Plant Biology 2008, 8:1 II
Received: 2 May 2008

Accepted: 7 November 2008

(C) 2008 Lin et al; licensee BioMed Central Ltd.

This is an Open Access article distributed under the terms of the Creative Commons Attribution License (http://creativecommons.org/licenses/by/2.0), which permits unrestricted use, distribution, and reproduction in any medium, provided the original work is properly cited.

\begin{abstract}
Background: CBF/DREB duplicate genes are widely distributed in higher plants and encode transcriptional factors, or CBFs, which bind a DNA regulatory element and impart responsiveness to low temperatures and dehydration.

Results: We explored patterns of genetic variations of $C B F I,-2$, and -3 from 34 accessions of Arabidopsis thaliana. Molecular population genetic analyses of these genes indicated that CBF2 has much reduced nucleotide diversity in the transcriptional unit and promoter, suggesting that CBF2 has been subjected to a recent adaptive sweep, which agrees with reports of a regulatory protein of CBF2. Investigating the ratios of $\mathrm{K}_{\mathrm{a}} / \mathrm{K}_{\mathrm{s}}$ between all paired $C B F$ paralogus genes, high conservation of the AP2 domain was observed, and the major divergence of proteins was the result of relaxation in two regions within the transcriptional activation domain which was under positive selection after $C B F$ duplication. With respect to the level of $C B F$ gene expression, several mutated nucleotides in the promoters of CBF3 and $-I$ of specific ecotypes might be responsible for its consistently low expression.
\end{abstract}

Conclusion: We concluded from our data that important evolutionary changes in $C B F I,-2$, and 3 may have primarily occurred at the level of gene regulation as well as in protein function.

\section{Background}

Genome duplication has been proposed as an advantageous path to evolutionary innovation and functional divergence $[1,2]$. Ohno's theory hypothesizes that duplicates are mostly silenced by degenerative mutations following duplication due to a redundancy in function. Although probabilities of duplicates acquiring novel and beneficial functions is lower than duplicates being silenced [3], the classical model of the fates of duplicate genes proposed by Ohno is coming under increasing scrutiny [4-6]. Preservation of duplicate genes is believed to be a common fate, particularly for genes that contain multiple regulatory regions, and thereby multiple copies of genes are maintained in the genome [7-10]. 
Duplicated proteins evolve for some time under relaxed functional constraints, after which functional divergence occurs when formerly neutral substitutions convey a selective advantage in a novel environment or genetic background [11]. Gene duplication is often followed by an elevated mutation rate $[11,12]$. Rapid duplicate gene evolution is caused by positive Darwinian selection or relaxation of the functional constraints in the redundant gene. Continuation of gene duplication leads to the formation of gene groups with high similarities in nucleotide and amino acid sequences. The majority of genes in higher organisms are members of multigene families or subfamilies [13]. Three potential evolutionary fates of duplicated genes have been suggested [1,14]: (A) Duplicated genes may be inactivated by the degenerative mutations and become nonfunctional (i.e., defunctionalization). (B) A duplicated gene may diverge to acquire a new function (i.e., neofunctionalization). (C) Degenerative mutations occur in each copy after duplication. Both genes may be altered in such a way that the combined activity of the two genes together fulfills the task of the ancestral gene in a complementary fashion (i.e., subfunctionalization) $[2,15]$.

CBF gene family, belongs to AP2/ERF (ethylene-responsive element-binding protein) superfamily, is considered as a result of gene duplication [16]. Functional redundancy and differentiation have been reported, however, evolutional aspect of this small gene family has not been investigated. The AP2/ERF superfamily is a large gene family of transcription factors and is defined by the AP2/ERF domain, which consists of 60 70 amino acids involved in DNA binding. CBF1/DREB1B, CBF3/DREB1A, and CBF2/ $D R E B 1 C$ are located in a tandem array within a region of $8.7 \mathrm{~kb}$ on chromosome 4 [[17], see Figure 1A] in Arabidopsis thaliana and were categorized into group IIIc in the ERF family [18]. The functions of $C B F 1,-2$, and -3 , which are induced by cold, have been shown to play crucial roles in the process of cold acclimation in Arabidopsis including low temperature-, and/or drought-stress-responsive gene expression $[17,19]$. CBF binds a DNA regulatory element, the C-repeat (CRT) dehydration-responsive element (DRE), which has a conserved core sequence of CCGAC, which imparts responsiveness to low temperatures and dehydration [20]. Comparisons of the transcriptomes indicated that $12 \%$ of the cold-responsive genes are confirmed members of the CBF regulon [21]. CBF4, which is located in chromosome 5, is involved in the dehydration response rather than to cold, and is less closely related to CBF1, -2, and -3 in phylogeny [22]. The amino acid sequences of the CBF1, -2 , and -3 proteins are highly similar, with approximately $88 \%$ identities and $91 \%$ similarities [17]. But do they have redundant functions? By comparing the effects of the overexpression of each $C B F$ gene in Arabidopsis, it was determined that, in fact, they induce expression of similar gene sets [16]. However, other molecular or physiological studies have indicated functional divergence among $C B F$ genes [23-26].

In this report, we attempted to characterize the molecular population genetics and gene expression of these three $C B F$ genes from 34 ecotypes, and to assess the roles of selective forces that have driven the divergence of these three duplicate genes. Usually molecular evolutionary approaches are used to study selection forces on gene sequences; here we have the opportunity to check whether the results from those methods are supported by many studies of the expression and function of $C B F$ genes. Our results, coupled with those from other published studies, indicate that functional divergence in $C B F 1,-2$, and -3 resulted from promoter polymorphisms as well as coding sequence variations.

\section{Results \\ Sequence polymorphisms of CBFI, -2, and -3 in Arabidopsis thaliana}

We obtained $C B F$ genomic sequences from 34 geographically distant ecotypes covering Europe, North America, Russia, North Africa, Japan, and India (see additional file 1). Substantial differences of polymorphic patterns were observed among the three CBFs. In the promoter region analyzed, CBF1 possessed the highest nucleotide diversity, $\pi[48]$, of 0.02749 , which was 25 -fold higher than that of CBF2 and 3.5-fold higher than that of CBF3. A similar trend was observed when another nucleotide diversity estimate, $\theta_{\mathrm{W}}[49]$, was examined (Table 1 ). The CBF1 promoter region also had the most abundant indels (26 regions of indels, 0.096 per site) compared to CBF2 (eight regions of indels, 0.077 per site) and CBF3's promoter regions ( 12 regions of indels, 0.013 per site). The distributions of indels are demonstrated in the additional file (see additional files 2, 3 and 4). Most polymorphic sites in $C B F 1$ 's promoter were phylogenetically informative (Figure $1 \mathrm{~B}$ ), and the promoter sequences after site 195 (see additional file $2 \mathrm{~A}$ ) seemed to be dominated by dimorphic polymorphism. Compared to $C B F 1$, most of the polymorphic sites in $C B F 2$ 's promoter were singletons, and the frequencies of informative sites and singletons were compatible in CBF3 (Figure 1D).

For CBF transcriptional units (TUs) the nucleotide diversity $(\pi)$ of CBF3 was highest $(0.00685)$ but close to that of CBF1 (0.00548), while the diversity of CBF2 was still the lowest (0.00264). However, the nucleotide diversity, $\pi$, of CBF2's TU was 2.4-fold higher than that of its promoter (Table 1). In the CBF coding regions, 11, 11, and 16 nonsynonymous substitutions were respectively found in CBF1, -2 , and -3 . Of the total 38 nonsynonymous substitutions, only three were located in AP2 domains (but outside the proposed alpha-helix motif) which are 
A Chromosome 4


Figure I

Genomic map and orientation of CBF's (A), and gene structures and polymorphism illustrations of CBFI (B), CBF2 (C) and CBF3 (D). (A): Promoter regions are represented by horizontal lines and transcriptional units (TUs) are indicated by boxes. Bold lines indicate sequenced promoter regions. Grey boxes indicated coding regions. The arrows above TUs represent direction of transcription. The starting and ending positions of CBF TU are indicated (M: mega base pair) based on AtDB Sequence Map. (B), (C), and (D): Promoter regions are represented by horizontal lines, and TUs are indicated by boxes (UTR: white boxes; coding region: labeled boxes). Lines and triangles above the gene structure respectively indicate polymorphisms and indels which are informative, and those below the gene structure indicate singletons; black circles are nonsynonymous substitutions; numbers next to the black circles represents number of nonsynonymous substitutions. Boxes with diamonds are nuclear localization signals; boxes with diagonal lines indicate the AP2 domain; gray boxes with diagonal lines are alpha-helix motifs; heavy gray boxes are transcriptional activation domains; dotted boxes are not functionally defined. Gray lines parallel to the gene structures represent long indel in non-coding regions, coding regions with amino acid deletions (CBFI) and a loss of complete open reading frame (CBF2). See "Materials and methods" and additional files 2, 3, 4. The scale bars indicate 100 bp.

responsible for DNA binding. Seven nonsynonymous substitutions were in the $\mathrm{N}$-terminal region where the function is unknown. No amino acid replacement was observed in the NLS. Twenty-eight nonsynonymous substitutions existed in transcriptional activation domains which were responsible for activation of CBF regulon genes. Ten of 11 amino acid displacements in CBF2 were singletons except for one in the $\mathrm{N}$-terminal region (Figure 1C).

Ecotypes Hi-0 and Bl-1 had lost ten amino acids in CBF1's transcriptional activation domain, but their open-reading frames were still present. A single adenine insertion was found in the CBF2 coding region of Kas-2. This insertion, located at the beginning of the transcriptional activation domain, led to an early nonsense stop codon in this reading frame, and 92 of $\sim 98$ residues of this domain had been lost. This C-terminal domain is necessary for activation of the expressions of downstream genes [50]. We believe that CBF2 in the Kas-2 ecotype has become a null allele. No indels were found in CBF3's coding regions. We sequenced CBF4 coding sequences in 34 ecotypes as well, and no indels were found. Two nonsynonymous substitutions were observed: one was a singleton and the other was informative. 
Table I: Nucleotide diversity of CBF s

\begin{tabular}{|c|c|c|c|c|c|c|c|c|c|c|}
\hline Region & $n$ & L (bp) & L-gaps & $\mathrm{H}$ & $\mathrm{S}$ & $\pi$ & $\pi_{\text {syn }}$ & $\pi_{\text {nonsyn }}$ & $\theta_{\mathrm{W}}$ & Tajima's D \\
\hline \multicolumn{11}{|c|}{ Promoters $^{\mathrm{a}}$} \\
\hline CBFI & 33 & 940 & 850 & 14 & 62 & 0.02749 & & & 0.01797 & $1.96 \#$ \\
\hline$C B F 2$ & 33 & 1190 & 1098 & 10 & 16 & 0.00109 & & & 0.00359 & $-2.32 * *$ \\
\hline CBF3 & 33 & 1298 & $|28|$ & 18 & 48 & 0.00788 & & & 0.00923 & -0.54 (N.S.) \\
\hline \multicolumn{11}{|l|}{ TUs } \\
\hline CBFI & 34 & 953 & 892 & 16 & 28 & 0.00548 & 0.01788 & 0.00221 & 0.00768 & -1.00 (N.S.) \\
\hline CBF2 & 34 & 945 & 833 & 12 & 25 & 0.00264 & $0.0074 I$ & 0.00183 & 0.00734 & $-2.23^{* *}$ \\
\hline CBF3 & 34 & 911 & 904 & 15 & 40 & 0.00685 & 0.01490 & 0.00319 & 0.01082 & - I.32 (N.S.) \\
\hline CBF4b & 34 & 675 & 675 & 3 & 8 & 0.00319 & 0.01183 & 0.00069 & 0.00290 & 0.30 (N.S.) \\
\hline
\end{tabular}

a One promoter region for each CBF was excluded because of long sequence indels (see "Materials and methods"); b Only CDS regions were analyzed (accession nos.: EF523160 EF523192); n, number of sequences analyzed; L, aligned sequence length; L-gaps, sequence length without missing data; $\mathrm{H}$, number of haplotypes; $\mathrm{S}$, number of segregated sites; $\pi$, nucleotide diversity estimated by pair-wise comparisons from all sites; $\pi_{\text {syn }}$, nucleotide diversity estimated from synonymous sites; $\pi_{\text {nonsyn }}$, nucleotide diversity estimated from nonsynonymous sites; $\theta_{W}$, nucleotide diversity estimated based on segregating sites from all sites. ${ }^{*} p<0.1 ; * p<0.05$; $* * p<0.0$ I; N.S., not significant.

\section{Distinct patterns of sequence polymorphisms were observed in different regions of CBF}

Tajima's $D$ test was marginally positive in the promoter of CBF1 ( $D=1.96,0.1<p<0.05$, Table 1$)$, however, after removing sites before site 195 (see additional file $2 \mathrm{~A}$ ), the $D$ value was significantly positive $(D=2.12, p<0.05)$. This suggests a signature of balancing selection dominating the majority of the CBF1 promoter. In the TU of $C B F 1$, the $D$ value was not significant. In contrast to $C B F 1$, both the promoter and TU of CBF2 had an excess of singletons, and Tajima's $D$ tests were significantly negative $(-2.32$ and -2.23 in the promoter and TU, respectively, $p<0.01)$. This indicates that the entire region of $C B F 2$ was favored by positive selection, and a beneficial allele has been fixed in the population. However, a significant negative $D$ value can also be the result of deriving low-frequency detrimental alleles or a bottleneck effect. The signature of positive selection of $C B F 2$ was confirmed by other tests and physiological data as described below. Unlike CBF1 and -2, neither the promoter nor TU was significant in CBF3.

We scanned different regions of $C B F$ promoters and TUs using sliding windows of Tajima's $D$ test to identify regions which deviated from neutral expectations. In the promoter of $C B F 1, D$ values after about site 550 (Figure $2 \mathrm{~A}$; the region after site 195 in additional file 2A) were positive, and there were three major regions carrying significantly positive $D$ values, especially in regions within 300 bp upstream of the TU. We identified no regions which significantly departed from 0 in the promoter of $C B F 2$, and no positive $D$ values were observed. In the $C B F 3$ promoter, the proportions of positive and negative regions were compatible. A significant positive region (near site 100, Figure 2A) was observed. Fluctuations in $D$ values in the TUs were smoother than those of the promoter (Figure $2 \mathrm{~B}$ ). The entire region of CBF2's TU had a negative $D$ value, and two regions located in the transcriptional activation domain were significantly negative (Fig- ure $2 \mathrm{~B}$ ). Most of the singletons in these two regions were nonsynonymous substitutions (Figure 1C). CBF1, -2 , and -3 differed in the N-terminal of the AP2 domain where the $D$ value was nearly significant in $C B F 1(D=1.92)$ and negative in $C B F 2$ and -3 . Although several informative polymorphisms in $C B F 1$ in this region contributed to the difference in $D$, they were synonymous substitutions.

We obtained four copies of $C B F$-like sequences from $A$. lyrata ssp.petraea. One was closest to At $C B F 3$, and three were similar to At CBF2. We found no homologs of $C B F 1$ in A. lyrata. A phylogenetic tree was constructed according to haplotypes of CBFs coding regions (Figure 3). Haplotypes of different $C B F$ were assorted well to the same lineage according to their $C B F$ classification and the orthologs CBFs (Al CBF2 $a / 2 b / 2 c$ and Al CBF3) from $A$. lyrata were just located as outgroups of their assigned lineages. The $C B F 1$ was probably duplicated from the common ancestor of CBF2/CBF3 before the speciation of A. thaliana and A. lyrata. (see Figure 3 and Table 3), but was lost in A. lyrata; however we could not exclude the possibility that the divergence of $C B F 1$ has evolved to such an extent that it could not be found, or the sample size (26 clones) was simply not large enough.

Fay and Wu's $H$, and other statistics which use orthologous sequences as outgroups were computed. To obtain evidence of a recent selective sweep, we used Fay and Wu's $H$ test, which measures an excess of derived high-frequency mutations to intermediate-frequency mutations. A negative significant $H$ indicates a recent selective sweep. The $H$ value for the CBF2 coding region was significant ( $p$ $=0.0015$, Table 2) but not significant in CBF3 ( $p=$ 0.0681 ). We used two statistics, $G_{\text {mean }}$ and $D_{\mathrm{KS}}$, to detect the heterogeneity of polymorphism-to-divergence ratios in the coding regions of CBF2 and -3. Sliding windows of the polymorphism-to-divergence ratios were also plotted (additional file 5). Fixation of an advantageous mutation 

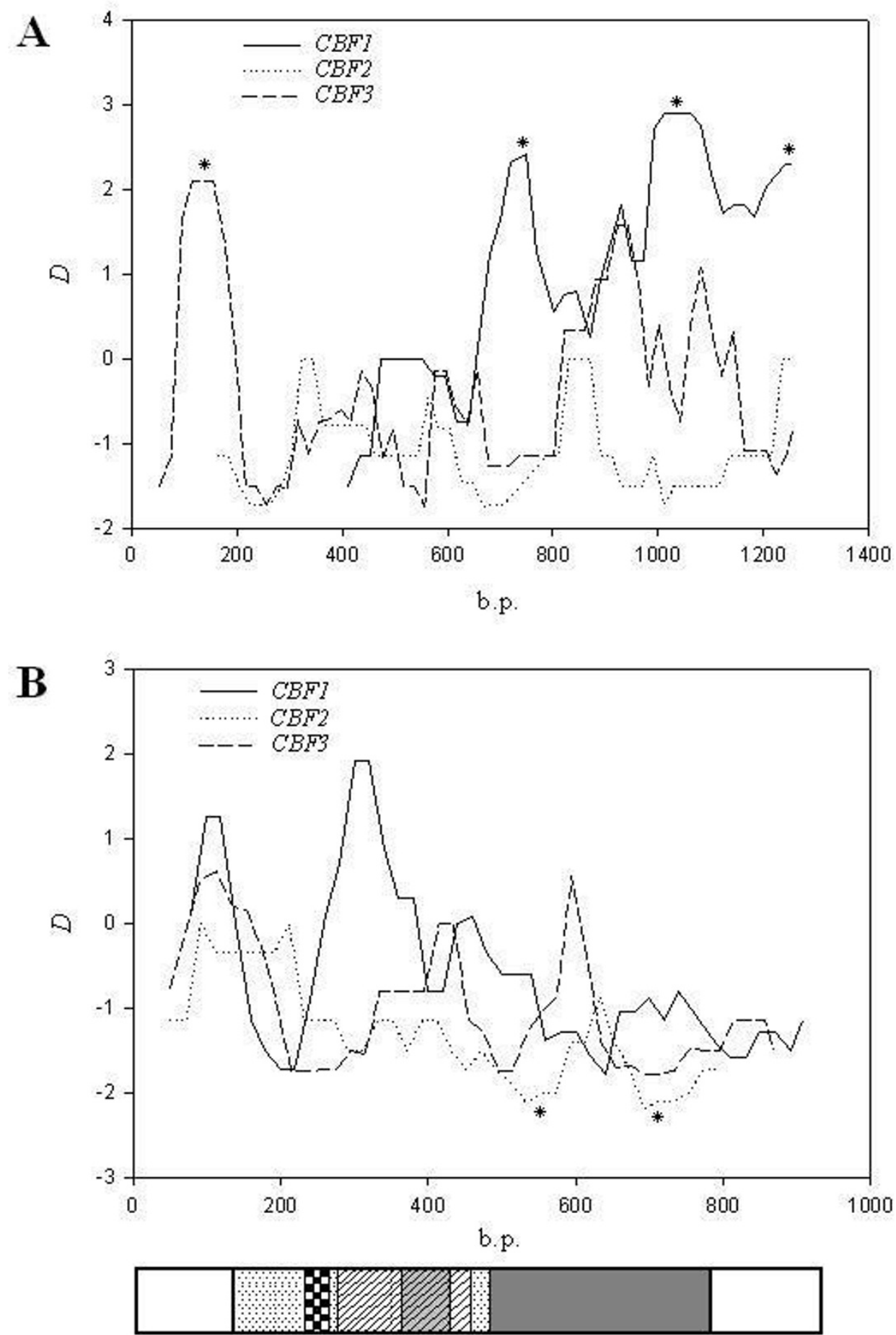

Figure 2

Sliding windows of Tajima's $D$ tests along promoters $(A)$ and transcriptional units (B) of CBFI, -2 , and -3 . Regions with significant positive or negative values $(p<0.05)$ are labeled with an asterisk $(*)$. The scale of the sliding window plot was adjusted for the promoter regions. The last ( $3^{\prime}$ end) sites of the promoter sequences of $C B F I,-2$, and -3 were placed in the same position. A transcriptional unit structure was placed under the corresponding nucleotide position. The window size is $100 \mathrm{bp}$, and the step size is $20 \mathrm{bp}$. An explanation of the boxes is given in the legend of Figure I. 


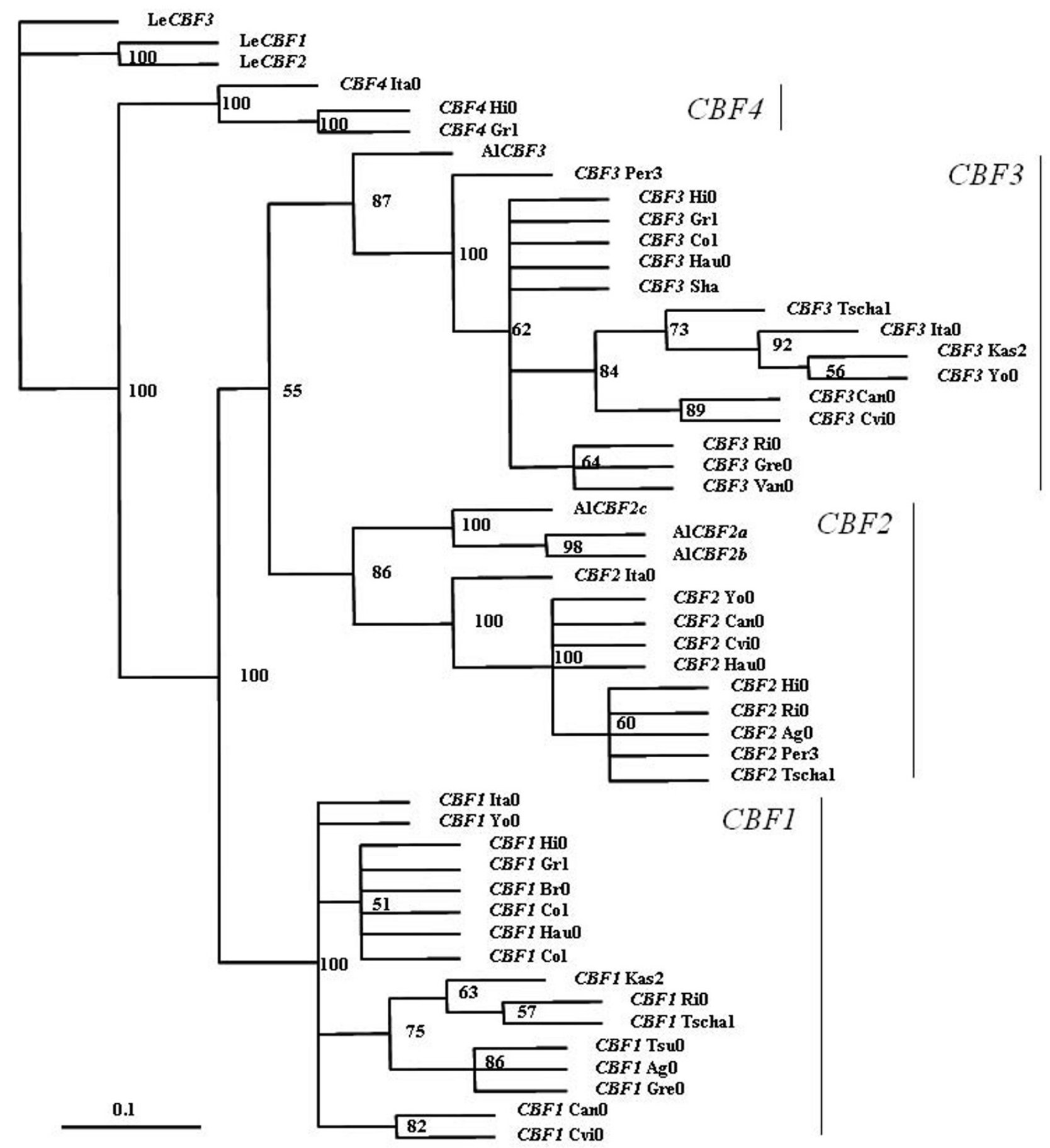

Figure 3

A phylogenetic tree of CBFs. The ecotypes used for phylogenetic tree construction were indicated after each $C B F$ taxon. The scale bar indicates 0.1 substitutions per site. The tree is unrooted. The groups of $C B F I,-2,-3$, and -4 was indicated by vertical bars. 
Table 2: Neutrality tests and tests of heterogeneity of polymorphisms to divergence using outgroup sequences

\begin{tabular}{llll}
\hline Region & Fay and Wu's $H$ & $\mathrm{G}_{\text {mean }}$ & $\mathrm{D}_{\mathrm{KS}}$ \\
\hline CBF2 & $-8.555\left(p=0.0015^{*}\right)$ & $0.036^{*}$ & $0.046^{*}$ \\
CBF3 & $-3.686(p=0.068 \mathrm{I})$ & $0.005^{* *}$ & 0.385
\end{tabular}

$p$ values of the $H$ statistics were generated by 10,000 replicate simulations; $G_{\text {mean }}$, mean sliding $G$ statistic; $D_{K S}$, Kolmogorov-Smirnov statistic; the highest $p$ values of $G_{\text {mean }}$ and $D_{K S}$ tests are reported in the table. $* p<0.05 ; * *<0.01$.

leads to a selective sweep which reduces the proportion of polymorphisms. A "valley" in the sliding window plot indicates a sweep, while balanced polymorphism leads to a "peak". $G_{\text {mean }}$ is most sensitive for detecting one or two peaks, and $\mathrm{D}_{\mathrm{KS}}$ is good at detecting a single low to high change in polymorphism [37]. The $\mathrm{G}_{\text {mean }}$ tests were significant for both $C B F 2$ and -3 , and $\mathrm{D}_{\mathrm{KS}}$ was significant for CBF2 (Table 2). The results indicated the presence of heterogeneity of polymorphism-to-divergence ratios in the coding region in CBF2 (and -3). In other words, localized selective sweeps were detected. Thus, the selective sweep event in CBF2 (especially in the coding region) gene was supported by four neutrality tests.

\section{Purifying selection acts on CBFs but different levels of divergence were observed among different domains}

We computed the ratios of $\mathrm{K}_{\mathrm{a}} / \mathrm{K}_{\mathrm{s}}$ between all paired $C B F$ paralogous and orthologous genes (Table 3 ). The ratio of $\mathrm{K}_{\mathrm{a}} / \mathrm{K}_{\mathrm{s}}$ indicates protein evolution where the values of $\omega$ of $>1,=1$, and $<1$ respectively indicate positive, neutral, and purifying selection. The ratios were comparable among different pair-wise comparisons. The lowest was 0.117 (CBF1 vs. CBF3) and the highest was 0.159 (CBF3 vs. $C B F 4)$. This indicates that strong purifying selection has been acting on $C B F$ s since their duplication and the speciation of $A$. thaliana and A. lyrata. Although CBF4 has been proven to be involved in the dehydration pathway and is not induced by cold [22], this gene's coding region did not diverge much from those of CBF1, -2 , or -3 based on $\mathrm{K}_{\mathrm{a}} / \mathrm{K}_{\mathrm{s}}$ ratios. To scan different divergences along the protein domains, we plotted sliding windows of $\mathrm{K}_{\mathrm{a}} / \mathrm{K}_{\mathrm{s}}$ for pairwise comparisons of CBF1, -2 , and -3 (Figure 4A), and between CBF1 (or -2 or -3 ) and CBF4 (Figure 4B). Substantial differences in divergences were observed. In the comparisons among $C B F 1,-2$, and -3 , the AP2 domain was the most conserved region especially in the region of the alpha-helix; the N-terminal regions upstream of the NLS had moderate ratios ranging from 0.3 to 0.7 ; and the transcriptional activation domain had the greatest divergence which contained two major divergent regions with ratios of 0.997 and 0.808 (Figure $4 \mathrm{~A}$ ). The higher conservation of the AP2 domain in $C B F 1 / 2 / 3$ indicates that members of regulons of each $C B F$ should be similar.
Compared to the divergence among cold-induced $C B F 1$, 2 , and -3 , the divergent region between dehydrationinduced CBF4 and CBF1, -2 , and -3 was unique. The AP2 domains were apparently conserved because there were some gaps in the N-terminal side of this domain. There is a region within the transcriptional activation domain which had $K_{a} / K_{s}$ ratios which were all > 1 (1.6 3.0), a signature of positive selection in protein evolution, in all three comparisons. The three highest $\mathrm{K}_{\mathrm{a}} / \mathrm{K}_{\mathrm{s}}$ peaks covered almost the same region (Figure 4B).

Positive selection in the transcriptional activation domain So far we have found that the most divergent regions are in the transcriptional activation domains of CBFs; however, the sliding window plots detected no region with a $\omega$ $\left(\mathrm{K}_{\mathrm{a}} / \mathrm{K}_{\mathrm{s}}\right.$ ratio) value of $>1$. This is because for sliding window plots, each window (of $50 \mathrm{bp}$ ) overlapped with the previous and following windows because the step size (10 bp) was smaller than the window size. These sizes of the window and overlapping windows might not have allowed us to detect smaller regions of positive selection or to have precisely located the divergent region. Herein we used a non-overlapping sliding window method to locate the existence of positive selection for six residues per window in the transcriptional activation domain.

Four regions within this domain were found to carry $\mathrm{K}_{\mathrm{a}} / \mathrm{K}_{\mathrm{s}}$ ratios of $>1$, including 1.193 in $C B F 1$ and -2 (amino acid residues 167 172 in Figure 5), 1.704 in CBF2 and -3 (amino acid residues 164 169), 30.51 (amino acid residues 137 142) and 22.08 (amino acid residues 162 167) in CBF1 and -3 comparisons (sliding window plot not shown). Compared to the sliding windows of Figure 4, this approach more-precisely located sites which may be under positive selection (Figure 5, regions with gray blocks). It is worth noting that except for the region labeled (with a $\omega$ value of $>1$ ) from amino residues 137 to 142 in the comparison of CBF1 and -3, three other regions almost completely overlapped (residue sites 162 172), and therefore actually two regions were under positive selection in this domain after $C B F$ duplication.

We used codeml in the PAML3.15 package [45] to estimate $\omega$ values among sites with different models. CBF2 sequences could not be computed because too many branches had collapsed after the bootstrap re-sampling. The results of all LRTs for CBF1 and -3 were significant (the highest $p$ value was 0.002 among all LRTs). M2a, M3, and M8 all identified a small proportion of protein (< $1 \%$ ) with $\omega$ values of $>1$, while most portions of CBF1 and -3 were under purifying selection. One positively selected site, $178 \mathrm{~T}$ (181 T in Figure 5; site703 in additional file 2B) was identified in CBF1, and two sites, $2 \mathrm{~S}$ (125 bp of CDS in additional file 4B) and 151E (152E in Figure 5; site572 in additional file 4B), were identified in 

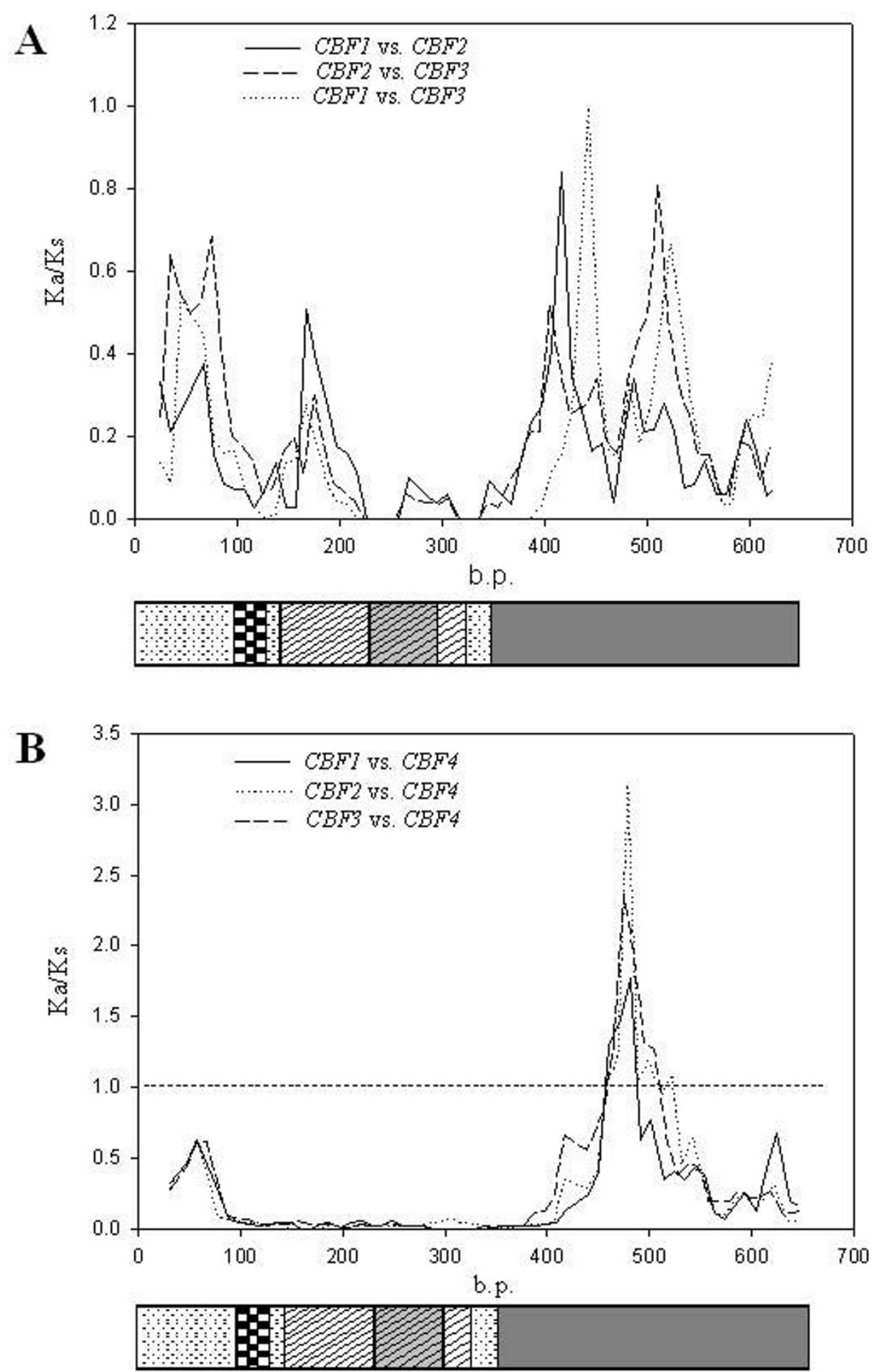

Figure 4

Sliding windows of $K_{a} / K_{s}$ ratios between pairs of $C B F I,-2$, and $-3(A)$ and between $C B F I$ (and -2 and - 3 ) and -4 (B). The nucleotide position was placed in correspondence to the coding region structure. The window size is $50 \mathrm{bp}$, and the step size is $10 \mathrm{bp}$. An explanation of the boxes is given in Figure I. 
Table 3: Evolutionary distances between paralogs and orthologs in non-coding and coding regions

\begin{tabular}{|c|c|c|c|c|}
\hline Region & $\mathrm{K}$ & $\mathrm{K}_{\mathrm{s}}$ & $\mathrm{K}_{\mathrm{a}}$ & $\mathrm{K}_{\mathrm{a}} / \mathrm{K}_{\mathrm{s}}$ \\
\hline Promoters ${ }^{a}$ (paralogs) & & N.A & N.A & N.A \\
\hline$C B F I$ vs. $C B F 2$ & $0.448 \pm 0.043$ & & & \\
\hline$C B F 2$ vs. $C B F 3$ & $0.360 \pm 0.035$ & & & \\
\hline$C B F I$ vs. $C B F 3$ & $0.501 \pm 0.045$ & & & \\
\hline \multicolumn{5}{|l|}{ UTR'b (paralogs) } \\
\hline$C B F I$ vs. $C B F 2$ & $0.212 \pm 0.041$ & & & \\
\hline CBF2 vs. $C B F 3$ & $0.156 \pm 0.033$ & & & \\
\hline CBFI vs. CBF3 & $0.266 \pm 0.047$ & & & \\
\hline CDS (paralogs) & N.A. & & & \\
\hline$C B F I$ vs. $C B F 2$ & & $0.408 \pm 0.069$ & $0.062 \pm 0.012$ & 0.152 \\
\hline CBF2 vs. $C B F 3$ & & $0.462 \pm 0.078$ & $0.068 \pm 0.014$ & 0.147 \\
\hline CBFI vs. CBF3 & & $0.546 \pm 0.093$ & $0.064 \pm 0.013$ & 0.117 \\
\hline CBFI vs. CBF4 & & $1.478 \pm 0.45 \mathrm{I}$ & $0.216 \pm 0.031$ & 0.146 \\
\hline CBF2 vs. CBF4 & & $1.728 \pm 0.654$ & $0.233 \pm 0.032$ & 0.135 \\
\hline CBF3 vs. CBF4 & & $1.456 \pm 0.431$ & $0.232 \pm 0.030$ & 0.159 \\
\hline \multicolumn{5}{|l|}{ CDS (orthologsc) } \\
\hline$A t C B F 2$ vs. $A / C B F 2$ & & $0.340 \pm 0.059$ & $0.048 \pm 0.012$ & 0.141 \\
\hline$A t C B F 3$ vs. $A I C B F 3$ & & $0.284 \pm 0.056$ & $0.045 \pm 0.011$ & 0.158 \\
\hline
\end{tabular}

a Regions containing 500 bp upstream from the transcriptional unit (TU) were analyzed; $K$, average sequence distance between promoter regions of $C B F s$; 'b the $5^{\prime}$ and $3^{\prime}$ untranslated regions (UTRs) were analyzed; $K_{s}$, average distance between CBFs estimated using synonymous sites; $K_{a}$, average distance between CBFs estimated using nonsynonymous sites; N.A., not allowed. c AICBF2 (accession nos.: EF523I93 EF523I95) and AICBF3 (accession no.: EF523196).

CBF3. Nine ecotypes carried $178 \mathrm{~S}$ instead of $178 \mathrm{~T}$ in $\mathrm{CBF} 1$, while in CBF2 and -3 , all ecotypes carried a serine residue at this site. Seven ecotypes carried $2 \mathrm{~S}$ instead of 2 $\mathrm{N}$, and seven ecotypes carried 151A instead of $151 \mathrm{E}$ in CBF3.

\section{Gene expression experiments identify several naturally knockout or knockdown of CBFs}

In CBF1, a 211-bp region in the 3'-end of promoter was substituted with a 261-bp insertion in Ita-0, and a 365-bp insertion was found just $10 \mathrm{bp}$ from the transcriptional initiation site in Co-1 (see additional file 2). CBF1 expression in Ita-0 was low, and the signal was still visible within

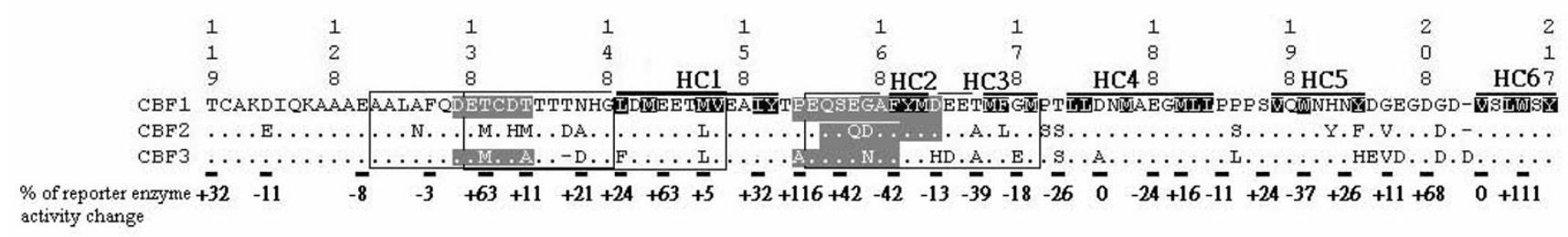

\section{Figure 5}

Sites of predicted positive selection in the transcriptional activation domains of CBFI, -2, and -3. The figures were modified according to Figure 3B of Wang et al. [50]. Ninety-eight amino acids of the CBFI C-terminal were aligned with the same domains of CBF2 and -3 (Col ecotype). The numbers above the upper panel are amino acid numbers of the protein sequence alignment. Six hydrophobic clusters are labeled by a line above each cluster, and hydrophobic residues within clusters are indicated by black blocks. Reporter constructs possessing an alanine-substituted CBFI activation domain were used to estimate the contribution of motifs or residues to transactivation activity. The alanine-substituted residues are indicated by black underlining below each site, and the percentages of changes in reporter enzyme ( $\beta$-galactosidase) activity related to the wildtype CBFI activation domain construct are indicated just below each underlined alanine substitution. Sequences boxed by rectangles indicate regions which had the highest $\mathrm{K}_{\mathrm{a}} / \mathrm{K}_{\mathrm{s}}$ ratios in the transcriptional activation domains in Figure $4 \mathrm{~A}$. Gray blocks covering amino acid sequences indicate regions in which the $\mathrm{K}_{\mathrm{a}} / \mathrm{K}_{\mathrm{s}}$ ratios exceed $\mathrm{I}$ using a non-overlapping sliding window method (i.e., the gray block covering residue sites 137 to 142 representing this region of six residues was detected in the comparison of $C B F I$ and -3 ). There are three pair-wise comparisons between $C B F I,-2$, and -3 , and four regions were labeled by gray blocks. 
the same expression scale (Figure 6), while Co-1 had no $C B F 1$ expression. Although $C B F 2$ in Cvi-0 had lost most of the sequenced promoter (the region of $1 \sim 1030 \mathrm{bp}$ was lost), CBF2 was still expressed at a low level. In CBF3 promoters, an 864-bp region was substituted with a 1798-bp insertion in Kas-2. The signal intensity of $C B F 3$ expression in Kas- 2 was $\sim 10^{-3}$ fold compared to those of the others. We believe the 1798-bp substitution removed all cis-elements from the Kas-2 CBF3's promoter.

In addition to naturally occurring mutants, we found that the expressions of $C B F 3$ in Cvi-0 and Co- 1 , and $C B F 1$ in Cvi-0 and Can- 0 after $4{ }^{\circ} \mathrm{C}$ treatment for $1.5 \mathrm{~h}$ were consistently low in different batches. However, gene expressions in most other ecotypes were not consistently high or low among batches (data not shown).

\section{Discussion}

\section{DNA sequence polymorphism}

The overall level of genome-wide polymorphism in Arabidopsis was estimated in the values between 0.06 and 0.07
$[51,52]$. The $\pi$ values of $C B F 3$ 's promoter, and $C B F 1$ and 3 's TUs are close to these two estimates. Altogether, CBF2 was the most highly conserved gene in the promoter region and TU of this small family, and CBF1 carried the highest nucleotide diversity in the promoter, the diversity of TU was compatible with that of the TU of CBF3. The nucleotide diversities of promoters of $C B F$ s were greater than those among TUs. This suggests that the promoters and TUs of CBFs have been subjected to distinct selection mechanisms and/or demographic histories. With respect to protein evolution, AP2 domains were very conservative compared to the transcriptional activation domains. This suggests that among CBFs in Arabidopsis, the DNA targeting abilities have not changed throughout their evolutionary history. The divergence, if any, of CBF biochemical functions should exist in transcriptional activation domains.

Different selection forces working on different CBF genes Selection has played a role in the maintenance of conservation in coding sequences among $C B F 1,-2$, and -3 in the

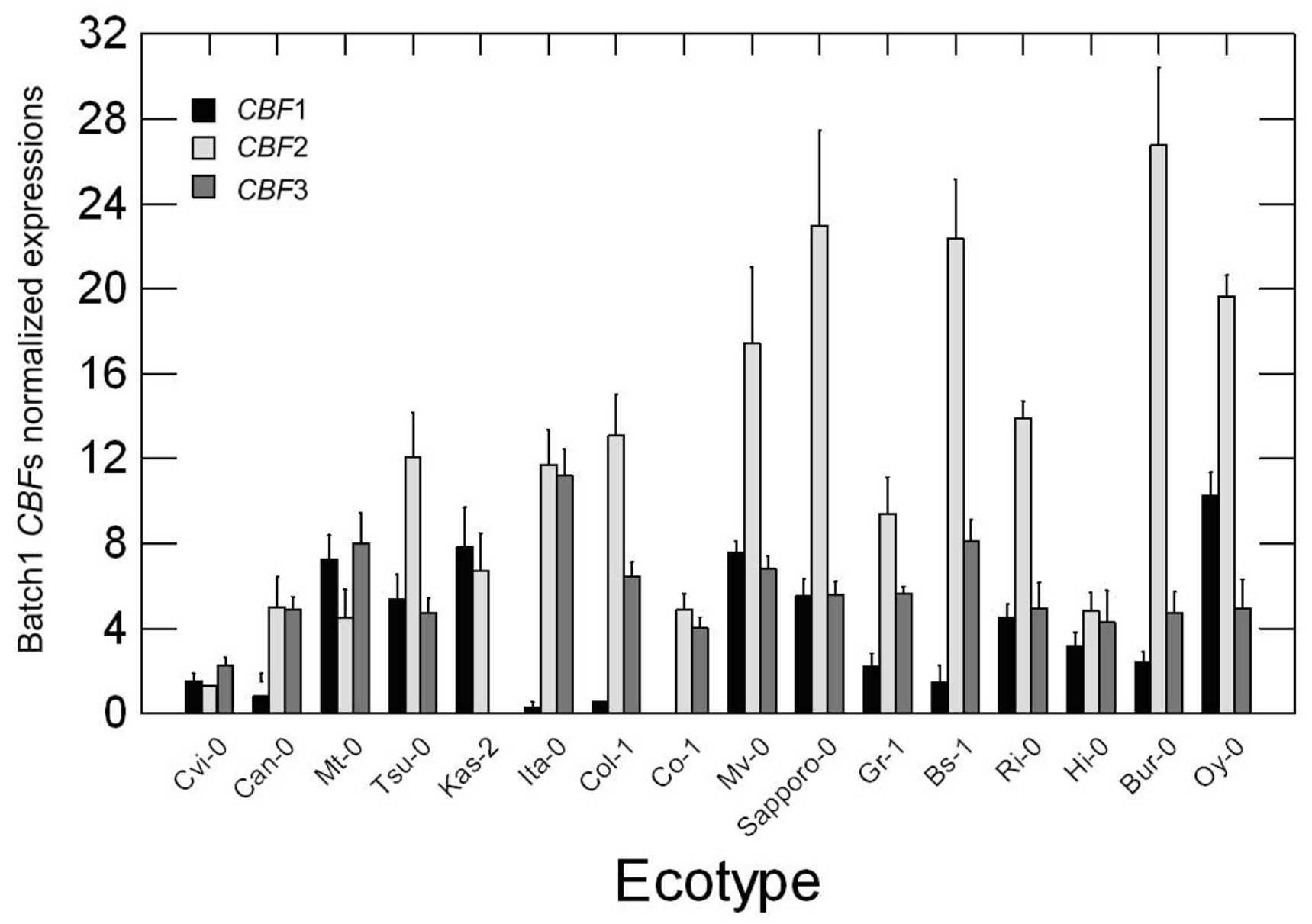

Figure 6

CBF expressions in each ecotype. Quantities of CBFI (dark bars) and -3 (grey bars) expressions were normalized to the ACTIN2 expression quantities of the same ecotypes. The ecotypes are placed in latitudinal order (left to right: south to north). 
form of purifying selection. As to protein evolution, these three duplicate genes are functionally constrained as evidenced by the $\mathrm{Ka} / \mathrm{Ks}$ ratios being much lower than the neutral expectation of 1 for both paralogs and orthologs (Table 3). The excess of low-frequency polymorphisms for CBF2 indicates that it is strictly constrained. The nucleotide variation in the CBF1 promoter was characterized by dimorphism indicating balancing selection, while the entire region of $\mathrm{CBF} 3$ did not deviate from neutral expectations.

However, a strong population structure and genome-wide deviation from the standard neutral model in A. thaliana were reported [51,52]. Nordberg et al. [51] suggested that the skewness of Tajima's $D$ of the A. thaliana genome was due to an excess of rare alleles and demographic processes. To reassess the influence of the population structure, we used the Mantel test (zt software [53]) to evaluate the existence of isolation-by-distance in our dataset. The promoters and transcriptional units of $C B F 1,-2$, and -3 were examined. The results from both regions of $C B F 2$ were not significant (promoter: $r=-0.055, p=0.345$; TU: $r=-0.031, p=0.556)$, but were significant in the promoters of CBF1 and $-3(r=0.146, p=0.037$; and $r=0.134$, $p$ $=0.046)$. It seems the results from Tajima's $D$ test of CBF2 are independent of the population structure. Nevertheless, the population structure is expected to push Tajima's $D$ toward positive values [51], and this effect may have partially contributed to the significantly positive $D$ of $C B F 1$ 's promoter. However, the dimorphism we observed was not related to the geographic distribution of ecotypes. The origins of maintaining such allelic dimorphism are still unclear. This pattern of allelic dimorphism is not unprecedented in Arabidopsis, having been reported for many loci including TFL1 [54]. It may be a result of the demographic history of this species complex [55].

We do think that $C B F 2$ played a unique role different from those of $C B F 1$ and -3 after gene duplication. It is possible that the regulatory function of $C B F 2$ was favored by positive selection which was detected in our dataset.

\section{Positive selection sites in the transcriptional activation domain}

The transcriptional activation domain of CBF1 was studied by Wang $e t$ al. [50], and six hydrophobic cluster motifs were identified by a computational analysis. They used a series of $\mathrm{GAL} 4_{\mathrm{DBD}} / \mathrm{CBF} 1_{\mathrm{AD}}\left(\mathrm{CBF} 1_{\mathrm{AD}}\right.$ : transcriptional activation domain) fusion constructs containing either nonsense codon introductions or alanine substitutions in $\mathrm{CBF} 1_{\mathrm{AD}}$ to assay their abilities to transactivate the GAL4responsive lac $Z$ reporter enzyme in yeast. Based on the high sequence identity and the fact that almost the same set of downstream genes can be activated by $C B F 1,-2$, and -3 , the mechanism of transcriptional regulation of $C B F$ regulons should be very similar. Thus, the functional dissection of this domain from CBF1 might also be the case in CBF2 and -3 .

The non-overlapping sliding window of $\mathrm{K}_{\mathrm{a}} / \mathrm{K}_{\mathrm{s}}$ suggests that two possible regions were favored by positive selection after gene duplication (Figure 5). These two regions are not located in the hydrophobic clusters 2, 3, and 4 which Wang et al. [50] considered to have been the most critical motifs in transactivation. In these two regions, four of six residues substituted by alanine led to substantial changes in reporter enzyme activities $(139 \mathrm{~T} \rightarrow \mathrm{A}, 162 \mathrm{P}$ $\rightarrow \mathrm{A}, 165 \mathrm{~S} \rightarrow \mathrm{A}$, and $169 \mathrm{~F} \rightarrow \mathrm{A}$, Figure 5). It was interesting to note that the activity increase of $162 \mathrm{P} \rightarrow \mathrm{A}$ was the highest among substitutions, and $169 \mathrm{~F} \rightarrow \mathrm{A}$ was also the greatest among those that led to activity decreases.

We want to see whether the positive selection site in the sequence agrees with the two regions mentioned above. With the site models of the codeml program, two positively selected amino acids were identified in the transcriptional activation domain as expected but not locate in the two regions identified by the sliding window of $\mathrm{K}_{\mathrm{a}} /$ $\mathrm{K}_{\mathrm{s}}$ method.

A decrease of $26 \%$ of reporter enzyme activity was observed when alanine was substituted for 181 T of CBF1, and in $\mathrm{CBF} 3,152 \mathrm{E}$ is in hydrophobic cluster 1 . When the same site in CBF1 was substituted by alanine $(152 \mathrm{E} \rightarrow \mathrm{A})$, a $63 \%$ increase in reporter activity was observed (Figure $5)$.

Hydrophobic residues within the functionally critical $\mathrm{HC} 2,-3$, and -4 of CBFs were found to be conserved among different ecotypes. Most positively selected regions of the activation domain identified in this paper are located outside of critical motifs. This difference is because the meaning of $\mathrm{K}_{\mathrm{a}} / \mathrm{K}_{\mathrm{s}}$ is different from that of hydrophobic cluster, the former emphasizes variation in amino acid replacement and the latter in no or low variation. It implies that the hydrophobic cluster is critical in the interaction with other activator and the two regions found in this study might involve in tuning the transcriptional activation.

\section{Ecotypes from lower latitude may accumulate mutations that reduce CBFs expressions}

Some variations we found for specific nucleotides of the promoters of $C B F 1$ and -3 were probably responsible for the low level of gene expression. CBF1 of Cvi-0 has four nucleotides which are unique among the ecotypes (nos. 1, 249, 469, and 541, see additional file 2), while Can-0 has two unique nucleotides (nos. 57 and 469). CBF3 of Cvi-0 of has four nucleotides which are unique among the ecotypes (nos. 338, 1017, 1167, and 1284 in additional file 
4). However, none of these nucleotides was located in the known cis-element of $C B F 1$ or -3 when screened by the PLANTCARE website [56].

It is interesting to note that singletons of the CBF1 promoters of Cvi-0 and Can-0 comprised 35\% (6/17) of the 34 accessions, and nucleotide mutations mainly occurred in accessions collected from lower latitudes (see additional file 2). Singletons of the CBF3 promoters of Cvi-0 and Co- 1 comprised $16 \%$ (5/31) of the 34 accessions, and nucleotide mutations also mainly occurred in lower latitudes (see additional file 4). Thus lower latitudes (probably indicating warmer-temperature environments) might allow accumulation of nucleotide mutations in the promoter regions.

\section{Evidence of functional diversification in CBFI, -2, and -3} In a study to test for benefits and costs (number of fruits) of cold tolerance, CBF2 and CBF3 overexpressers showed costs of cold tolerance and no fitness benefits in control and cold environments. CBF1-overexpressing plants showed no fitness costs of cold tolerance in the control environment and showed a marginal fitness benefit in the cold environment [24]. A CBF2 knockout mutant, cbf2, had a higher capacity to tolerate freezing than wild-type ones which was correlated with stronger and more-sustained expressions of $C B F 1$ and $C B F 3$ in the mutant. They concluded that $C B F 2$ is a negative regulator of $C B F 1$ and CBF3 [25], and CBF2 but not CBF1 and CBF3 was found as a gene contributed to a major freezing QTL locus [26]. Moreover, in contrast to CBF2, CBF1 and CBF3 positively regulate cold acclimation by activating the same subset of CBF-target genes [57]. These studies indicated functional changes have occurred at the level of CBF proteins.

Ecotype Kas-2 carries a CBF3 knockout allele and a null allele $C B F 2$ gene due to a single insertion in the coding region. At first, we predicted that $C B F 1$, which is the only functional $C B F$ induced by cold, in this ecotype would be highly expressed to compensate for the double-mutant effect. However, this prediction was not correct. This is also evidence that CBF1, -2 , and -3 are not strictly functional equivalents.

Evolutionarily important changes have also occurred at the level of gene regulation in CBF1, -2 , and -3 in addition to protein function. Chinnusamy et al. [23] identified a mutant, ICE1 (inducer of CBF expression 1), that results in the $C B F 3$ gene no longer being induced in response to low temperature, but which has little effect on cold induction of CBF1 and CBF2. CBFs are subjected to different temporal regulation during cold acclimation. Recently, Novillo et al. [57] further supported that CBF1 and CBF3 are regulated in a different way then $C B F 2$. Although subfunctionalization of the coding region has been detected, subfunction in the promoter is also a process leading to separate expression control of $C B F s$.

\section{Conclusion}

In summary according to sequence analyses, we demonstrate that different selection forces have been working on the promoter and coding regions of $C B F 1,-2$, and -3. After $C B F$ duplication, CBF2 experienced selective sweeps in the promoter and coding regions, and this may have resulted in its unique functional divergence which differs from those of $C B F 1$ and -3 . The promoter of $C B F 1$ has a signature of balancing selection, while CBF3 did not deviate from neutrality. $C B F$ promoters share less similarity than do the coding regions, indicating they are differentially regulated or have been shaped by different evolutionary forces. There is evidence of positive selection in both the CBF1 and -3' transcriptional activation domains within each domain and between them. CBF1 and - 3 were shown to activate same regulons but need to work cooperatively [57], and they are regulated by different upstream factors [23]. The function of the progenitor CBF gene has not been determined to date. Nevertheless, it seems that the complements of $C B F 1$ and -3 are under subfunctionalization, while $C B F 2$, with a different regulatory role, might be a case of neofunctionalization.

\section{Methods \\ Growth conditions, genomic polymerase chain reaction (PCR), and sequencing}

All ecotype resources are listed in the supplementary materials (see additional file 1 ). The seeds were chilled at $4{ }^{\circ} \mathrm{C}$ in the dark for 5 days before being grown in soil medium (Bio-mix TTing Substratum, Moerdijk, Nederland). Seeds of A. lyrata ssp.petraea (Plech, Bavaria, Germany) were chilled for more than 2 weeks. The seedlings were grown at $22^{\circ} \mathrm{C}$ under a 16 -h light/ 8 -h dark photoperiod with a light intensity of $\sim 100 \mu \mathrm{E} / \mathrm{m}^{2} / \mathrm{s}$. For the gene expression study, three-week old seedlings were treated at $4{ }^{\circ} \mathrm{C}$, for $1.5 \mathrm{~h}$ under fluorescent lights at $40 \mu \mathrm{E} / \mathrm{m}^{2} \mathrm{~s}$. To avoid the effects of circadian clock behavior of $C B F \mathrm{~s}$, we always started the chilling treatment at 13:00 (ZT4, [27]). After cold treatment, leaves from five individuals were combined as one sample for each ecotype and were immediately frozen in liquid nitrogen. Three independent trials were performed. The interval between trials was $3 \sim 4$ weeks.

Genomic DNA was extracted following a modified protocol according to Doyle and Doyle [28] and stored at $20^{\circ} \mathrm{C}$. Primers (see additional files 6 and 7 for primer sequences and PCR conditions) were designed according to the ecotype Col genomic sequence in The Arabidopsis Information Resource (TAIR) [29] using the Primer3 program [30]. We used the PreMix2.0 (TOPBIO, Taipei, Taiwan) PCR reagent mix for all PCR reactions. The PCR 
products were run on $1 \%$ agarose gels, and the desired bands were excised for purification and direct sequencing using a Taq Dye Dideoxy Terminator Cycle Sequencing Kit (Applied Biosystems, Foster city, CA, USA) and an ABI 3730 sequencer (Applied Biosystems). Vector NTI Suite 9 Contig-Express (Invitrogen, Karlsruhe, Germany) was used for sequence assembly and chromatogram inspection.

The CBF3 genes in the various ecotypes were first sequenced. To avoid PCR and sequencing errors, two independent PCR samples for each ecotype were separately sequenced. In addition to the primers used in the PCRs, additional primers were designed and used in sequenced regions with ambiguous signals. No sequence difference was observed among any two independent $C B F 3$ sequence replicates, and therefore we performed one PCR for CBF1 and -2. Primers in the PCR reactions for $C B F 1$ and -2 were used for sequencing, and additional primers were also used to sequence ambiguous regions (the second PCRs were performed for sequencing ambiguous regions). To isolate $C B F$ orthologs from $A$. lyrata, primers were designed to amplify the coding regions. The genomic-PCR products from A. lyrata were purified by gelelution. After the PCR fragments were ligated into the vector, pGEM ${ }^{\circledast}-\mathrm{T}$ Easy (Promega, Madison, WI, USA), the constructs were transformed to competent DH5- $\alpha$ cells (RBC Bioscience, Taipei, Taiwan). Twenty-six colonies were selected for sequencing. Each copy of the $C B F$ orthologs was determined based on at least three clones. In this research, because the sequences of CBF4 were used as the outgroup for certain sequence and phylogenetic analyses mentioned below, only the coding regions of CBF4 were determined.

Sequence data from this article have been deposited with the GenBank Data Libraries under accession nos: EF522962 EF523192, EF523193 EF523195, and EF523196. Genomic sequences of Col of AT4G25470 (CBF2), AT4G25480 (CBF3), AT4G25490 (CBF1), and AT5G51990 (CBF4) were retrieved from TAIR.

\section{Sequence analysis}

Sequences were aligned using CLUSTAL X [31]. Certain insertions were manually removed after sequence alignments to maximize alignable regions prior to calculating the polymorphism parameters. In the analysis of promoter sequences, $C B F 1,-2$, and -3 respectively of Ita- 0 , Cvi-0, and Kas-2 were excluded. In the promoter sequences from Ita-0's $C B F 1$ and Kas-2's $C B F 3$, there was a region substituted by a sequence from another part of the genome. Most parts of the $C B F 2$ promoter region had been lost in Cvi-0 in the region we surveyed. The promoter of Co-1 CBF1 was included in our analysis after removing a 365-bp insertion near the site of transcriptional initiation. In the analysis of nucleotide polymor- phism of the transcriptional unit, the CBF2 sequences from Ita- 0 and Kas-2 were included after removing their insertions in the $3^{\prime}$ untranslated region (UTR). In Ita-0, a long insertion ( $>1100 \mathrm{bp}$ ) and a GGGAAA sequence ( 5 bp before the insertion) were removed from the 3'UTR, but we did not finish sequencing this insertion. The remaining 3'UTR sequence of Ita-0 after this insertion was treated as missing data. Coding sequences of CBF1 of Hi0 and $\mathrm{Bl}-1$ and CBF2 of Kas-2 were not analyzed in the $\mathrm{K}_{\mathrm{a}} /$ $\mathrm{K}_{\mathrm{s}}$ calculation to avoid loss of polymorphism information in these regions due to gaps that led to amino acid deletions (Hi-0 and Bl-1) and frame-shifts (Kas-2).

Sequence divergences between orthologs and paralogs were estimated using MEGA3.1 [32]. The evolutionary distance, $\mathrm{K}$, between CBF promoters (500 bp upstream from the TU) were computed using the Kimura 2-paramater substitution model, and gaps were treated as missing data. Distances between orthologous coding sequences, and between paralogous sequences were computed from synonymous $\left(\mathrm{K}_{\mathrm{s}}\right)$ and nonsynonymous sites $\left(\mathrm{K}_{\mathrm{a}}\right)$ by the Nei-Gojobori method [33]. All standard errors of divergence distances were determined using 500 bootstrap replicates.

The nucleotide diversity, Tajima's $D$ test [34], Fay and Wu's $H$ [35], and a sliding window analysis were carried out using DNASP4.10 [36]. The window size for the promoter and TU analyses was $100 \mathrm{bp}$ with a step size of 20 bp. The window size for the $\mathrm{K}_{\mathrm{a}} / \mathrm{K}_{\mathrm{s}}$ sliding window plots was $50 \mathrm{bp}$ with a step size of $10 \mathrm{bp}$. In order to identify fluctuations in $\mathrm{K}_{\mathrm{a}} / \mathrm{K}_{\mathrm{s}}$ more precisely in the transcriptional activation domains, we used a sliding window approach in which the window and step sizes were both $18 \mathrm{bp}$. This allowed non-overlapping window scanning for six residues per window. Fay and Wu's $H$ was computed using $A$. lyrata $C B F$ orthologs as outgroups. The heterogeneity of polymorphisms to fixed differences was computed using DNA Slider1.11 [37]. Only sequences from silent sites were considered. For $\mathrm{G}_{\text {mean }}$ and $\mathrm{D}_{\mathrm{KS}}$ statistics, $\mathrm{R}=2,4,8$, 16 , and 32 were simulated with 1000 replicates. The highest $p$ value of each statistic is reported.

A CBF phylogenetic tree was constructed by the Neighborjoining method [38] under the HKY85 [39] model based on the coding sequences using PAUP*4.10 [40]. One thousand bootstrap replicates were performed, and $>50 \%$ frequencies are shown. The bootstraps values are given at each node. One sequence (from one ecotype) of each haplotype of coding sequences was used. $C B F$ homologs from Lycopersicon esculentum, Le CBF1, -2, and -3 (GenBank

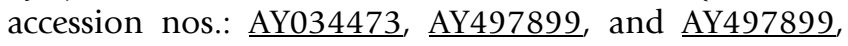
respectively), were used as outgroups.

In addition to locating the sequence region being under selection by the sliding window approach, we want to fur- 
ther find the sites are positively selected. Because it is impossible that positive selection operates on every lineage (at different times) and every codon has the same strength, Yang developed statistical tests based on the maximum-likelihood method [41-43] to estimate specific lineages or codons which are under positive selection. The value of $\omega$ is allowed to vary among sites (codons), and sites under positive selection can be identified by site models. In the one-ratio model ( $\mathrm{M} 0)$, all lineages and sites have the same value of $\omega$. In the site models (M1a, $\mathrm{M} 2 \mathrm{a}, \mathrm{M} 3, \mathrm{M} 7$, and M8), classes of $\omega$ are allowed according to different assumptions $[41,43]$. These models were designed as hierarchal relationships, and log likelihood values obtained by M0 (one-ratio), M1a (nearly neutral), M2a (positive selection), M3 (discrete), M7 (beta), and M8 (beta and selection) were compared using likelihood ratio tests (LRTs). The significances of $\mathrm{M0}$ vs. M3, M1a vs. M2a, and M7 vs. M8 were estimated [44]. Codeml in the PAML3.15 package [45] was used to detect specific sites (or codons) which were positively selected. Phylogenetic trees of $C B F 1,-2$, and -3 were constructed by maximumlikelihood using the HKY85 [39] model, and > 50\% branching topology was retained after 1000 bootstrap replicates. Trees were constructed according to full-length (promoter and TU) sequences using PAUP*4.10 [40]. $C B F 1,-2$, and -3 respectively in Ita- 0 , Cvi- 0 , and Kas- 2 were abandoned when estimating positively selected codons because of their long sequence indels.

The promoter sequences of $C B F$ genes were analyzed using TCS1.21 software [46]. Alignment gaps were treated as missing data. The promoters of $C B F 1,-2$, and -3 were sorted according to haplotypes. There are 15, 15, and 18 haplotypes of the CBF1,-2, and -3 promoters, respectively. Promoter haplotypes of ecotypes with higher frequencies were selected, and totally 16 ecotypes were chosen for the $C B F$ expression analysis.

\section{RNA extraction and complementary (c)DNA synthesis}

RNA was isolated using the REzol ${ }^{\mathrm{TM}} \mathrm{C} \& \mathrm{~T}$ (Protech, Taipei, Taiwan) reagent following the manufacturer's protocol. RNA was dissolved in DEPC-treated sterile water. After checking the RNA integrity on a $1 \%$ agarose gel, $4 \mu \mathrm{l}$ (2 $\mu \mathrm{g}$ ) of RNA was used to synthesize cDNA for each 20- $\mu$ l reaction volume by Superscript III-RT with oligo-d $\mathrm{d}_{12-18}$ primers according to the manufacturer's protocol (Invitrogen). Each sample was diluted1/20-fold.

\section{Real-time PCR analysis}

Different promoter haplotypes were used for real-time PCR analysis. The promoter haplotypes were sorted by TCS [46], and 16 ecotypes were chosen (covering most of promoter haplotypes) for expression analysis.
Three primer pairs were designed to measure the expressions of $C B F 1,-2$, and -3 (see additional file 8 for primer sequences). The primer pair for $C B F 1$ expression measurements, CBF1LP212/CBF1RP369, was designed according to the sequence of the $\mathrm{N}$-terminal coding region; the length of the CBF1 PCR fragment was $158 \mathrm{bp}$. For CBF2 measurements, the primer pair, CBF2LP744/CBF2RP913, was designed according to sequences of the C-terminal coding region and 3'UTR; the length of the CBF2 PCR fragment was 170 bp. For CBF3, the primer pair, CBF3LP38/CBF3RP196, was designed from the sequence of the 5'UTR and N-terminal of the coding region; the length of the CBF3 PCR fragment was $159 \mathrm{bp}$. All primers were designed in regions specific to each $C B F$ but which were conserved among the ecotypes used. Therefore, to consider the specificity and annealing temperatures of the primer pairs, we could not design them all according to the 3 '-end of sequences since reverse transcription may produce truncated cDNA. For each cDNA sample, the expressions of $C B F 1,-2$, and -3 were all analyzed. Primers of the housekeeping genes ACTIN2 (AT3G18780) and ACT2LP602/ACT2RP739, were designed to span a 78-bp 2nd intron. This allowed us to detect genomic DNA contamination in our cDNA samples. The presence of two or more PCR products, with different lengths and GC contents, was detected by melting kinetics, which were measured in every plate for every sample. The annealing temperature $(\mathrm{Tm})$ of all primer pairs was close to $60^{\circ} \mathrm{C}$. After sequencing the PCR products amplified by different primer pairs, we confirmed that our amplifications were specific to each CBF and ACTIN2, but not other highly similar members.

We used the iCycler iQ real-time detection system (BioRad Laboratories, Hercules, CA, USA) to quantify gene expression. Fifteen-microliter reaction mixtures (per well) contained $7.5 \mu$ l of the two-fold enzyme mix ( $\mathrm{iQ}^{\mathrm{TM}}$ SYBR Green Supermix, Bio-Rad), $1.9 \mu \mathrm{l}$ deionized/sterile water, $0.3 \mu \mathrm{l}$ of each primer ( $300 \mathrm{nM}$ final concentration), and 5 $\mu \mathrm{l}$ diluted cDNA $(\sim 0.1 \mu \mathrm{g})$. We used the same PCR protocol for all CBF1, -2 , and -3 and ACTIN2 primer pairs. The PCR protocol was $95^{\circ} \mathrm{C} 3$ min for hot-start polymerase (one cycle), $95^{\circ} \mathrm{C} 30 \mathrm{~s}, 60^{\circ} \mathrm{C} 30 \mathrm{~s}$, and $72^{\circ} \mathrm{C} 15 \mathrm{~s}$ for 40 cycles. After each PCR we performed melting kinetics to monitor the specificity of the CBF and ACTIN2 amplifications.

Standard curves of specific CBFs and ACTIN2 were established in every plate to quantify the relative expression levels (the formula of the standard curve quantification method was based on the ABI PRISM 7700 Sequence Detection System user bulletin \#2). For each cold-treated cDNA sample, four sample repeats were measured in every plate for both the CBFs and ACTIN2. The data of real-time PCR were analyzed using iCycler iQ optical sys- 
tem software 3.1 (Bio-Rad). Baseline cycles were set to two ten cycles, and the threshold position of the fluorescence was set to 30. Expressed quantities of each $C B F$ of the ecotypes were normalized to ACTIN2 quantities of the same ecotype. PCR efficiencies of different primer pairs were calculated according to Rasmussen's method [47]. The average PCR efficiency was $>97 \%$.

\section{Authors' contributions}

YHL designed and performed all analyses, analyzed the data and drafted the manuscript. SYH contributed analysis tools. PYH, CLH and CNW participated in the experiment. YCC provided the seed stock of Arabidopsis thaliana. TPL was the principal investigators. All authors contributed to the final manuscript, read and approved it.

\section{Additional material}

\section{Additional file 1}

Information of ecotypes and seed stocks used in our research. Click here for file

[http://www.biomedcentral.com/content/supplementary/1471-

2229-8-111-S1.doc]

\section{Additional file 2}

CBF1 sequence polymorphism. Description: (A) promoter region (accession nos.: EF522995 EF523027); (B) transcriptional unit (TU) region (accession nos.: EF522962 EF522994); UTR, untranslated region; CDS, coding sequence. Only polymorphic sites are listed. The number of each polymorphic site is listed according to its position in the sequence alignment, and the greater the number is, the closer to the transcriptional starting site it is. Dots represent nucleotides and indels identical to the Col reference; deletions and insertions related to the Col sequence are indicated by minus (-) and plus $(+)$ symbols, respectively. The sequences of indels of more than $1 \mathrm{bp}$ (base pair) are not shown. The length of each indel is listed at the bottom. The bold lines above the TU indicate the regions of the 5'UTR, CDS, and 3'UTR. Regions with long sequence deletions are blank; the ABRC (Arabidopsis Biological Resource Center) [73] stock numbers are next to the abbreviation of ecotypes. The promoter region from site 680 to the end (261 bp) was substituted by a 211-bp insertion in Ita- 0 .

Click here for file

[http://www.biomedcentral.com/content/supplementary/14712229-8-111-S2.tiff]

\section{Additional file 3}

CBF2 sequence polymorphism. Description: (A) promoter region (accession nos.: EF523061 EF523093); (B) transcriptional unit (TU) region (accession nos.: EF523028 EF523060). The symbols are the same as those in Figure 1. From site 648 to 665 in the promoter, there were different numbers of TA and TAA simple sequence repeats among ecotypes, but this region was absent from Kas-2 (from site 604). In our sequenced promoter region, site 1 1030 was absent from Cvi-0. The sequence of Ita0 after site 837 in the TU was excluded due to an insertion (of unknown length).

Click here for file

[http://www.biomedcentral.com/content/supplementary/1471-

2229-8-111-S3.tiff]

\section{Additional file 4}

CBF3 sequence polymorphism. Description: (A) promoter region (accession nos.: EF523127 EF523159); (B) transcriptional unit (TU) region (accession nos.: EFEF523094 EF523126). The symbols are the same as those in Figure 1. A 864-bp region (from site 420) was replaced by a 1798-bp insertion in the promoter of Kas-2.

Click here for file

[http://www.biomedcentral.com/content/supplementary/14712229-8-111-S4.tiff]

\section{Additional file 5}

S4: Sliding window plots of CBF2 and -3 polymorphism-to-divergence ratios. Description: Distribution of polymorphism to divergence ratios along CBF2 and CBF3 coding sequences using orthologus CBFs from A. lyrata ssp. petrea as outgroups. Each window contained 10 variable sites. Click here for file

[http://www.biomedcentral.com/content/supplementary/14712229-8-111-S5.tiff]

\section{Additional file 6}

Primer sequences used in genomic PCR and sequencing. Click here for file [http://www.biomedcentral.com/content/supplementary/14712229-8-111-S6.doc]

\section{Additional file 7}

Genomic PCR conditions.

Click here for file

[http://www.biomedcentral.com/content/supplementary/14712229-8-111-S7.doc]

\section{Additional file 8}

Primer sequences used in the real-time PCR.

Click here for file

[http://www.biomedcentral.com/content/supplementary/14712229-8-111-S8.doc]

\section{Acknowledgements}

This study was supported by grants from the National Taiwan University (97R0066-35) and the National Science Council (NSC94-2262 I-B-034-00 I and NSC95-2262I-B-034-00I) Executive Yuan, Taiwan. The authors are grateful for the donation of seed stocks of $A$. lyrata ssp. petraea and $A$. thaliana from Dr. Maria Clauss and Dr. Chiang, Yu-Chung, respectively.

\section{References}

I. Ohno S: Evolution by gene duplication. Springer-Verlag, Heidelberg, Germany; 1970.

2. Force A, Lynch M, Pickett FB, Amores A, Yan YL, Postlethwait J: Preservation of duplicate genes by complementary, degenerative mutations. Genetics 1999, 1 5 1: 153 I-1545.

3. Lynch M, Force AG: The origin of interspecific genomic incompatibility via gene duplication. Am Nat 2000, 156:590-605.

4. Massingham T, Davies IJ, Liò P: Analysing gene function after duplication. Bioessays 200I, 23(1 0):873-876.

5. Duarte JM, Cui L, Wall PK, Zhang Q, Zhang X, Leebens-Mack J, Ma $\mathrm{H}$, Altman N, dePamphilis W: Expression pattern shifts following duplication indicative of subfunctionalization and neofunctionalization in regulatory genes of Arabidopsis. Mol Biol Evol 2006, 23:469-478.

6. Yang X, Tuskan GA, Cheng ZM: Divergence of the Dof gene families in poplar, Arabidopsis, and rice suggests multiple modes of gene evolution after duplication. Plant Physiol 2006, 1 42:820-830. 
7. Ohta T: Simulating evolution by gene duplication. Genetics 1987, I | 5:207-2I3

8. Clark AG: Invasion and maintenance of a gene duplication. Proc Natl Acad Sci USA 1994, 91 : 2950-2954.

9. Nowak MA, Boerlijst MC, Cooke J, Smith JM: Evolution of genetic redundancy. Nature 1997, 388:167-17I.

10. Wagner A: Redundant gene functions and natural selection. Evol Biol 1999, I 2:1-16.

1I. Zhang J, Rosenberg HF, Nei M: Positive Darwinian selection after gene duplication in primate ribonuclease genes. Proc Natl Acad Sci USA 1998, 95:3708-37I3.

12. Bielawski JP, Yang Z: Positive and negative selection in the DAZ gene family. Mol Biol Evol 200I, 18:523-529.

13. Hughes AL: Adaptive evolution of genes and genomes. Oxford University Press, Oxford, UK; 1999.

14. Lynch M, Conery JS: The evolutionary fate and consequences of duplicate genes. Science 2000, 290: II5I-II55.

15. Hughes $A L$ : The evolution of functionally novel proteins after gene duplication. Proc R Soc Lond Ser B 1994, 256: I 19-124.

16. Gilmour SJ, Fowler SG, Thomashow MF: Arabidopsis transcriptional activators CBFI, CBF2, and CBF3 have matching functional activities. Plant Mol Biol 2004, 54:767-78I.

17. Gilmour SJ, Zarka DG, Stockinger EJ, Salazar MP, Houghton JM, Thomashow MF: Low temperature regulation of the Arabidopsis CBF family of AP2 transcriptional activators as an early step in cold-induced COR gene expression. Plant $J$ 1998, 16:433-442.

18. Nakano T, Suzuki K, Fujimura T, Shinshi H: Genome-wide analysis of ERF gene family in Arabidopsis and rice. Plant Physiol 2006 , | 40:4 | I-432.

19. Liu Q, Kasuga M, Sakuma Y, Abe H, Miura S, Yamaguchi-Shinozaki K, Shinozaki K: Two transcription factors, DREBI and DREB2, with an EREBPIAP2 DNA binding domain separate two cellular signal transduction pathways in drought- and low-temperature-responsive gene expression, respectively, in Arabidopsis. Plant Cell 1998, I0:139|-|406.

20. Yamaguchi-Shinozaki K, Shinizaki K: A novel cis-acting element in an Arabidopsis gene is involved in responsiveness to drought, low-temperature or high-salt stress. Plant Cell 1994, 6:25I-164

21. Fowler S, Thomashow MF: Arabidopsis transcriptome profiling indicates that multiple regulatory pathways are activated during cold acclimation in addition to the CBF cold response pathway. Plant Cell 2002, I4:1675-1690.

22. Haake V, Cook D, Riechmann JL, Pineda O, Thomashow MF, Zhang JZ: Transcription factor CBF4 is a regulator of drought adaptation in Arabidopsis. Plant Physiol 2002, 130:639-648.

23. Chinnusamy V, Ohta M, Kanrar S, Lee BH, Hong X, Agarwal M, Zhu JK: ICEI: a regulator of cold-induced transcriptome and freezing tolerance in Arabidopsis. Genes Dev 2003, 17:1043-1054.

24. Jackson MW, Stinchcombe JR, Korves TM, Schmitt J: Costs and benefits of cold tolerance in transgenic Arabidopsis thaliana. Mol Ecol 2004, 13:3609-36I5.

25. Novillo $F$, Alonso JM, Ecker JR, Salinas J: CBF2/DREBIC is a negative regulator of CBFI/DREBIB and CBF3/DREB IA expression and plays a central role in stress tolerance in Arabidopsis. Proc Natl Acad Sci USA 2004, 101:3985-3990.

26. Alonso-Blanco C, Gomez-Mena C, Llorente F, Koornneef M, Salinas J, Martínez-Zapater JM: Genetic and molecular analyses of natural variation indicate CBF2 as a candidate gene for underlying a freezing tolerance quantitative trait locus in Arabidopsis. Plant Physiol 2005, 139:1304-1312.

27. Fowler SG, Cook D, Thomashow MF: Low temperature induction of Arabidopsis CBFI, 2, and 3 is gated by the circadian clock. Plant Physiol 2005, 137:961-968.

28. Doyle JJ, Doyle JL: Isolation of plant DNA from fresh tissue. Focus 1990, 12:13-15.

29. TAIR, The Arabidopsis Information Resource [http:// www.arabidopsis.org]

30. Rozen S, Skaletsky H]: Primer3 on the WWW for general users and for biologist programmers, Bioinformatics Methods and Protocols: Methods in Molecular Biology. Edited by: Krawetz S, Misener S. Humana Press, Totowa, NJ; 2000:365-386.

31. Thompson JD, Gibson TJ, Plewniak F, Jeanmougin F, Higgins DG: The Clustal_X windows interface: flexible strategies for multiple sequence alignment aided by quality analysis tools. Nucleic Acids Res 1997, 25:4876-4882.
32. Kumar S, Tamura K, Nei M: MEGA3: integrated software for molecular evolutionary genetics analysis and sequence alignment. Brief Bioinform 2004, 5: 150-163.

33. Nei M, Gojobori T: Simple methods for estimating the numbers of synonymous and nonsynonymous nucleotide substitutions. Mol Biol Evol 1986, 3:418-426.

34. Tajima F: Statistical method for testing the neutral mutation hypothesis by DNA polymorphism. Genetics 1989, I 23:585-595.

35. Fay JC, $\mathrm{Wu} \mathrm{Cl}$ : Hitchhiking under positive Darwinian selection. Genetics 2000, 155: /405-I4I3.

36. Rozas J, Sanchez-DelBarrio JC, Messeguer X, Rozas R: DnaSP, DNA polymorphism analyses by the coalescent and other methods. Bioinformatics 2003, 19:2496-2497.

37. McDonald JH: Improved tests for heterogeneity across a region of DNA sequence in the ratio of polymorphism to divergence. Mol Biol Evol 1998, 15:377-384.

38. Saitou N, Nei M: The neighbor-joining method: A new method for reconstructing phylogenetic trees. Mol Biol Evol 1987 , 4:406-425.

39. Hasegawa M, Kishino H, Yano T: Dating the human-ape splitting by a molecular clock of mitochondrial DNA. J Mol Evol 1985, 22:160-174.

40. Swofford D: PAUP*: phylogenetic analysis using parsimony (*and other methods). In Version 4 Sinauer Associates, Sunderland, MA; 2002.

4I. Nielsen R, Yang Z: Likelihood models for detecting positively selected amino acids and applications to the HIV-I envelope gene. Genetics 1998, I48:929-936.

42. Yang Z: Likelihood ratio tests for detecting positive selection and application to primate lysozyme evolution. Mol Biol Evol 1998, 15:568-573.

43. Yang Z, Nielsen R, Goldman N, Pederson AMK: Codon-substitution models for heterogeneous selection pressures among sites classes. Genetics 2000, 155:431-449.

44. Bielawski JP, Yang Z: Maximum likelihood methods for detecting adaptive evolution after gene duplication. I Struct Funct Genom 2003, 3:201-2I2.

45. Yang Z: PAML: a program package for phylogenetic analysis by maximum likelihood. Comput Appl BioSci 1997, 13:555-556.

46. Clement M, Posada D, Crandall K: TCS: a computer program to estimate gene genealogies. Mol Ecol 2000, 9:1657-1660.

47. Rasmussen R: Quantification on the LightCycler:Rapid Cycle Real-time PCR, Methods and Applications. Edited by: Meuer S, Wittwer C, Nakagawara K. Springer Press, Heidelberg; 2001:21-34.

48. Nei M: Molecular evolutionary genetics. Columbia University Press, New York; 1987.

49. Watterson GA: Number of segregating sites in genetic models without recombination. Theor Populat Biol 1975, 7:256-276.

50. Wang Z, Triezenberg SJ, Thomashow MF, Stockinger EJ: Multiple hydrophobic motifs in Arabidopsis CBFI COOH-terminus provide functional redundancy in transactivation. Plant Mol Biol 2005, 58:543-559.

5I. Nordborg M, Hu TT, Ishino Y, Jinal J, Christopher T, Zheng H, Bakker E, Calabrese P, Gladstone J, Goyal R, Jakobsson M, Kim S, Morozov Y, Padhukasahasram B, Plagnol V, Rosenberg NA, Shah C, Wall JD, Wang J, Zhao K, Kalbfleisch T, Schulz V, Kreitman M, Bergelson J: The pattern of polymorphism in Arabidopsis thaliana. Plos Biol 2005, 3: 1289-1299.

52. Schmid KJ, Ramos-Onsins S, Ringys-Becktein H, Weisshaar B, Mitchell-Olds T: A multilocus sequence survey in Arabidopsis thaliana reveals a genome-wide departure from a neutral model of DNA sequence polymorphism. Genetics 2005, 169:1601-1615

53. ZT software [http://www.psb.ugent.be/ erbon/mantel]

54. Olsen KM, Womack A, Garrett AR, Suddith JI, Purugganan MD: Contrasting evolutionary forces in the Arabidopsis thaliana floral developmental pathway. Genetics 2002, 160:164I-1650.

55. Sharbel T, Haubold B, Mitchell-Olds T: Genetic isolation by distance in Arabidopsis thaliana: biogeography and postglacial colonization of Europe. Mol Ecol 2000, 9:2109-2118.

56. PLANTCARE [http://bioinformatics.psb.ugent.be/webtools/plant care/html]

57. Novillo F, Medina J, Salinas ]: Arabidopsis CBFI and CBF3 have a different function than CBF2 in cold acclimation and define different gene classes in the CBF regulon. Proc Natl Acad Sci USA 2007, 104:21002-21007. 